\title{
Determinantes do controle da pressão arterial em homens assistidos na atenção primária à saúde
}

\author{
Control of the determinants of blood pressure in assisted men in \\ primary health care \\ Rosimery Cruz de Oliveira Dantas* \\ Neir Antunes Paes* \\ Ana Tereza Medeiros Cavalcanti da Silva* \\ Vitor Engrácia Valenti** \\ Jose Alfredo Ordenes Mora*** \\ Joana Zambrano Chambrone* * \\ Luiz Carlos de Abreu**** \\ Maria do Carmo Andrade Duarte de Farias*
}

\begin{abstract}
Resumo
A Hipertensão Arterial é uma condição clínica multifatorial com uma prevalência no Brasil de 30\% em adultos maiores de 20 anos e de 35,8\% nos homens, que estão mais expostos a fatores de risco e frequentam menos os serviços de saúde. Nosso objetivo é investigar o controle da pressão arterial de homens hipertensos atendidos no Serviços de Saúde da Atenção Primária em dois municípios Paraibanos. Este estudo foi comparativo com abordagem quantitativa, análise descritiva, aplicando-se regressão logística. Foi verificado que a maioria dos homens hipertensos nos municípios estudados não está sendo acompanhada pelos serviços e apresenta pressão arterial não controlada. São maiores de 60 anos, de raça não branca, estão em sobrepeso/obesidade, tabagistas e não etilistas. As variáveis que mantêm relação com o controle da pressão são raça, sobrepeso/obesidade e sedentarismo. Concluímos que a hipertensão é um grave problema de saúde pública que requer de todos (usuários, profissionais e gestores) mudança de atitude no enfrentamento desse problema. Para o controle da pressão arterial se faz necessário o acompanhamento do usuário pelas equipes dos serviços de saúde. É necessário intensificar ações focadas nas atividades físicas e alimentação saudável, bem como vincular os homens aos serviços de saúde, promovendo uma captação precoce, uma reorganização na forma operacional de atendimento, registro, horário de consulta e o fortalecimento das ações de educação em saúde.
\end{abstract}

Palavras-chave: Atenção primária à saúde. Fatores de risco. Hipertensão. Saúde do homem.

\begin{abstract}
Hypertension is a multifactorial clinical condition with a prevalence in Brazil of $30 \%$ in adults over 20 years and $35.8 \%$ in men, who are more exposed to risk factors and attend fewer the health services. The objective of this research was to investigate the control of blood pressure in hypertensive men, assisted by the Primary Health Care Services in two cities in the state of Paraíba and to identify the relationship between modifiable risk factors and sociodemographic factors with the control of blood pressure levels. This comparative study with a quantitative approach, descriptive analysis, applying logistic regression. It was verified that most hypertensive men in the cities studied, it is not being monitored by the authorities and has uncontrolled blood pressure. They are over 60 years, of non-white race, overweight / obese, smokers and non-drinkers. The variables that keep relation with pressure control are race, overweight / obesity and physical inactivity. We conclude that hypertension is a serious public health problem that requires all users, professionals and managers to change their attitude in tackling this problem. To control blood pressure, it is necessary the monitoring of the users by the teams of health services. It is also necessary to intensify actions focused on physical activities and healthy eating, as well as link the men to health services, thus promoting early diagnosis, a reorganization in operating the attendance, record, consultation time and the strengthening of health education activities.
\end{abstract}

Keywords: Primary health care. Risk factors. Hypertension. Men's Health.

DOI: $10.15343 / 0104-7809.20164002249256$

\footnotetext{
*Universidade Federal de Campina Grande- CFP/UACV, Campina Grande, PB, Brasil.

** Faculdade de Ciências e Tecnologia, UNESP, Presidente Prudente, SP, Brasil.

***Faculdade de Biociências, UNESP, Rio Claro, SP, Brasil

****Faculdade de Medicina do ABC, Santo André, SP, Brasil.

Os autores declaram não haver conflitos de interesse.
} 


\section{INTRODUÇÃO}

As mudanças ocorridas no perfil epidemiológico da população ao longo do tempo estão diretamente relacionadas aos padrões de vida, hábitos e costumes que os indivíduos vêm adotando em prol das exigências do mundo contemporâneo. Nessa busca, os sujeitos muitas vezes se expõem a situações de riscos, vulnerabilidade e sofrimentos que contribuem para a reincidência de doenças já erradicadas, ao surgimento de novas e ao aumento das doenças crônicas não transmissíveis (DCNT), dentre elas a Hipertensão Arterial Sistêmica (HAS), principal fator de risco modificável para as doenças cardiovasculares (DCV) e, combinada aos riscos alimentares, desponta como o segundo fator de risco mais importante para a carga total de doenças ${ }^{1,2}$. Destaca-se que o grupo masculino apresenta maior fator de risco para as $\mathrm{DCV}^{3}$.

A HAS é definida como condição clínica multifatorial e caracterizada por níveis elevados e sustentados de pressão arterial (PA), associando-se frequentemente a alterações funcionais e/ou estruturais dos órgãos-alvo e alterações metabólicas. Sua prevalência na população brasileira adulta (>20 anos) está acima de $30 \%$, pontuando $35,8 \%$ nos homens e $30 \%$ em mulheres. Acomete aproximadamente $25 \%$ da população mundial, com uma projeção estimada de $60 \%$ de aumento nos casos da doença em 2025 $5^{1,4}$.

Possui uma forte relação com fatores de risco sociodemográficos e modificáveis e, no grupo masculino, não é raro encontrar associação com o tabagismo, etilismo, idade avançada e sobrepeso/obesidade ${ }^{2,5,6}$. Dessa realidade destaca-se a necessidade de acompanhamento, monitoramento e controle deste agravo para esse contingente populacional, que requer além de ações profissionais, uma adequada adesão ao serviço por parte do grupo masculino, como uma atitude global em relação à própria saúde ${ }^{7}$.

Informações adicionas acerca da HAS em regiões específicas do Brasil colaboram para a implementação de novos estudos terapêuticos e ensaios clínicos com a intenção de controlar essa DCV.
Considerando que a HAS é fator de risco para o desenvolvimento de DCV e alvo de ações das políticas públicas de saúde, este estudo teve como principal objetivo investigar o controle da Pressão Arterial, de homens hipertensos, assistidos pelos Serviços de Saúde da Atenção Primária em dois municípios Paraibanos, identificando a relação existente entre os fatores de risco modificáveis e os sociodemográficos com o controle dos níveis pressóricos.

\section{MÉTODO}

Tratou-se de um estudo comparativo entre dois municípios paraibanos entre os anos de 2009 e 2012, com análise analítica. Foram utilizados dados secundários da base de dados da pesquisa desenvolvida por $\mathrm{Paes}^{8}$, cujo objetivo foi avaliar a efetividade no controle da hipertensão arterial sistêmica e associação com fatores de risco comparando o desempenho do Programa de Saúde da Família e de Unidades Básicas de Saúde de municípios do Estado da Paraíba, sendo realizado nos dois maiores municípios do Estado da Paraíba: João Pessoa (JP) e Campina Grande (CG). A amostra obtida para a pesquisa de Paes $(2008)^{8}$, teve como unidade primária as Unidades de Saúde (conglomerados) e como unidade secundária os usuários cadastrados no HIPERDIA em 2006/2007 (sorteio sistemático), adotandose um Nível de Confiança de 95\%, a=5\% e $\mathrm{e}=3,5 \%$.

Para esse estudo utilizou-se a população masculina do estudo de $\mathrm{Paes}^{8}$, e para definir a sua representatividade adotou-se o indicador proporção populacional, para se criar um intervalo de confiança (IC) de 99\% a partir da diferença entre as duas proporções populacionais (população de cadastrados e amostra do estudo), obteve-se um IC de - 0,007 e 0,127 , resultando, portanto em uma amostra representativa dos hipertensos cadastrados e acompanhados na atenção primária a saúde nos municípios de CG e JP.

Foi utilizado como critério de exclusão a 
não participação do indivíduo em qualquer um dos anos do estudo (internação, mudança ou óbito). Esse estudo apresentou como limitação o tamanho da amostra, haja visto o cálculo da pesquisa principal não ter sido realizado para contemplar isoladamente os sexos e sim um grupo, e outra limitação foi o momento da realização da aferição da pressão, que mesmo sendo um estudo de coorte, só ocorria uma vez a cada ano.

A variável dependente utilizada foi o controle da pressão arterial e os dados sociodemográficos e fatores modificáveis foram utilizados como variáveis independentes no modelo de regressão logística e por apresentarem inúmeras variações foram dicotomizados em: Tipo de Usuário (não acompanhado e acompanhado), Situação conjugal (vive só e vive acompanhado), Escolaridade (analfabeto/ alfabetizado e escolarizado/graduado), Raça auto-referida (não branca e branca), Ocupação (com ocupação e sem ocupação), Renda $(<2$ salários e $\geq 2$ salários), Faixa etária ( $<60$ anos e $\geq$ 60 anos). Sedentarismo, sobrepeso/obesidade, tabagismo e etilismo, foram dicotomizadas em sim e não.

Adotou-se um IC de 95,0\% e nível de significância de 5\% para o Odds Ratio. A associação entre as variáveis foi verificada através do teste Qui-quadrado (X2) com nível de significância de 5\% utilizando o SPSS versão 13.0.

A pressão arterial (PA) foi dicotomizada em controlada ( $1=$ sucesso $)$ e não controlada $(0$ = não sucesso), segundo a recomendação das VI Diretrizes Brasileiras de hipertensão ${ }^{2}$, cuja pressão controlada (PAC) apresenta valores pressóricos sistólicos $<140 \mathrm{mmHg}$ e diastólicos $<90 \mathrm{mmHg}$. Valores superiores determina pressão arterial não controlada (PANC).

A condição de acompanhamento dos usuários foi referenciada pelas $\mathrm{V}$ Diretrizes Brasileiras de hipertensão ${ }^{9}$ como aquele paciente com três ou mais consultas no ano, com a PA registrada no prontuário médico. Adotou-se, por tratar-se de uma coorte, como paciente acompanhado aquele que apresentou três anos consecutivos de acompanhamento e como não acompanhado aquele com pelo menos um ano sem acompanhamento.

Este trabalho foi aprovado pelo Comitê de Ética em Pesquisa do Centro de Ciências da Saúde da Universidade Federal da Paraíba sob protocolo no. 576671/2008-8, estando de acordo com a Resolução 466/12 do Conselho Nacional de Saúde ${ }^{10}$ e todos os pacientes leram e assinaram o Termo de Consentimento Livre e Esclarecido.

\section{RESULTADOS}

Utilizou-se uma amostra de 75 homens para o município de JP e 69 para o município de CG, encontrou-se um perfil sociodemográfico dos usuários hipertensos (Tabela 1) com características semelhantes nos dois municípios, na maioria $>60$ anos, que vive com alguma companhia, com certa escolaridade, de raça auto-referida não branca, sem ocupação e, com renda superior a dois salários mínimos.

$\mathrm{Na}$ condição de acompanhamento dos usuários, os municípios de JP e CG apresentaram grande parte de usuários não acompanhados, pois tinham menos de três consultas médicas registradas no prontuário ao ano.

Deste percentual mais do que a metade apresentou PANC. A Tabela 2, revela fatores de risco modificáveis com influência sobre o controle da PA, em que observamos em homens dos grupos JP e CG de acordo com o número de tabagistas, não etilistas, sedentários e com sobrepeso/obesidade.

A Tabela 3 apresenta, a partir do ajuste do modelo de regressão logístico, que as variáveis raça e sobrepeso/obesidade, por atenderem aos valores estabelecidos evidenciaram uma associação significativa com o controle da PA. Para o município de CG, como nenhuma variável apresentou significância, utilizou-se apenas a variável sedentarismo, selecionada pelo teste Pearson $X^{2}$. Essa variável manteve associação com o controle da pressão ao nível de significância. 
Tabela 1 - Tipo de acompanhamento da pressão arterial dos homens hipertensos atendidos na atenção primária a saúde segundo variáveis sociodemográficas selecionadas dos municípios de João Pessoa e Campina Grande - Paraíba, 2009.

\begin{tabular}{|c|c|c|c|c|c|c|c|c|c|}
\hline \multirow{3}{*}{ Variáveis } & & \multicolumn{4}{|c|}{ João Pessoa } & \multicolumn{4}{|c|}{ Campina Grande } \\
\hline & & \multirow[b]{2}{*}{$\mathbf{n}$} & \multicolumn{2}{|c|}{ PANC } & \multicolumn{2}{|l|}{ PAC } & \multicolumn{2}{|c|}{ PANC } & AC \\
\hline & & & $\%$ & $\mathbf{n}$ & $\%$ & $\mathbf{n}$ & $\%$ & $\mathbf{n}$ & $\%$ \\
\hline \multirow{2}{*}{$\begin{array}{l}\text { Tipo } \\
\text { Usuário }\end{array}$} & Não acompanhado & 29 & 64,4 & 20 & 66,7 & 24 & 55,8 & 17 & 65,4 \\
\hline & Acompanhado & 16 & 35,6 & 10 & 33,33 & 19 & 44,2 & 9 & 34,6 \\
\hline \multirow[t]{2}{*}{ Sit. Conjugal } & Vive só & 5 & 11,1 & 0 & 0,0 & 5 & 11,6 & 2 & 7,7 \\
\hline & Vive acompanhado & 40 & 88,9 & 30 & 100,0 & 38 & 88,4 & 24 & 92,3 \\
\hline \multirow[t]{2}{*}{ Escolaridade } & Analfa/Alfabetizado & 13 & 28,9 & 12 & 40,0 & 15 & 34,9 & 8 & 30,8 \\
\hline & Escolar/Graduado & 32 & 71,1 & 18 & 60,0 & 28 & 65,1 & 18 & 69,2 \\
\hline \multirow[t]{2}{*}{ Raça } & Não Branca & 35 & 77,8 & 18 & 60,0 & 27 & 62,8 & 13 & 50,0 \\
\hline & Branca & 10 & 22,2 & 12 & 40,0 & 16 & 37,2 & 13 & 50,0 \\
\hline \multirow[t]{2}{*}{ Ocupação } & Com ocupação & 2 & 4,4 & 3 & 10,0 & 21 & 48,8 & 9 & 34,6 \\
\hline & Sem ocupação & 43 & 95,6 & 27 & 90,0 & 22 & 51,2 & 17 & 65,4 \\
\hline \multirow[t]{2}{*}{ Renda } & <2 salários & 17 & 37,8 & 7 & 23,3 & 20 & 46,5 & 9 & 34,6 \\
\hline & 2 salários e + & 28 & 62,2 & 23 & 76,7 & 23 & 53,5 & 17 & 65,4 \\
\hline \multirow[t]{2}{*}{ Faixa Etária } & $<60$ anos & 15 & 33,3 & 12 & 40,0 & 19 & 44,2 & 9 & 34,6 \\
\hline & 60 anos $\mathrm{e}+$ & 30 & 66,7 & 18 & 60,0 & 24 & 55,8 & 17 & 65,4 \\
\hline
\end{tabular}

Fonte: Base de dados da pesquisa realizada em 2011, Paes (2008; 2009)

Analfa=analfabeto; Escolar= escolarizado

Tabela 2 - Tipo de acompanhamento da pressão arterial dos homens hipertensos atendidos na atenção primária a saúde segundo fatores de risco modificáveis dos municípios de João Pessoa e Campina Grande, 2011.

\begin{tabular}{|c|c|c|c|c|c|c|c|c|c|}
\hline \multirow{3}{*}{ Variáveis } & & \multicolumn{4}{|c|}{ João Pessoa } & \multicolumn{4}{|c|}{ Campina Grande } \\
\hline & & \multirow[b]{2}{*}{$\mathbf{n}$} & \multicolumn{2}{|c|}{ PANC } & PAC & \multicolumn{2}{|c|}{ PANC } & \multicolumn{2}{|c|}{ PAC } \\
\hline & & & $\%$ & $\mathbf{n}$ & $\%$ & $\mathbf{n}$ & $\%$ & $\mathbf{n}$ & $\%$ \\
\hline \multirow[t]{2}{*}{ Tabagismo } & Sim & 9 & 20,0 & 6 & 20,0 & 26 & 60,5 & 12 & 46,2 \\
\hline & Não & 36 & 80,0 & 24 & 80,0 & 17 & 39,5 & 14 & 53,8 \\
\hline \multirow[t]{2}{*}{ Etilismo } & Sim & 6 & 13,3 & 6 & 20,0 & 9 & 20,9 & 6 & 23,1 \\
\hline & Não & 39 & 86,7 & 24 & 80,0 & 34 & 79,1 & 20 & 76,9 \\
\hline \multirow[t]{2}{*}{ Sedentarismo } & Sim & 23 & 51,1 & 14 & 46,7 & 33 & 76,7 & 15 & 57,7 \\
\hline & Não & 22 & 48,9 & 16 & 53,3 & 10 & 23,3 & 11 & 42,3 \\
\hline \multirow[t]{2}{*}{ Sp/Obesidade } & Sim & 28 & 62,2 & 12 & 40,0 & 24 & 55,8 & 15 & 57,7 \\
\hline & Não & 17 & 37,8 & 18 & 60,0 & 19 & 44,2 & 11 & 42,3 \\
\hline
\end{tabular}

Fonte: Base de dados da pesquisa realizada em 2011, Paes $(2008 ; 2009)$

PANC- Pressão Arterial não controlada; PAC- Pressão Arterial controlada; Sp- Sobrepeso 
Tabela 3 - Distribuição das variáveis do modelo de regressão logística binária para os homens hipertensos atendidos na atenção primária a saúde nos municípios de João Pessoa e Campina Grande, 2011.

\begin{tabular}{lccccc}
\hline \multicolumn{1}{c}{ Variável } & Coeficiente & Significância & OR & \multicolumn{2}{c}{ I.C 95,0\% } \\
& & & Menor & Maior \\
\hline & & João Pessoa & & 0,987 \\
\hline Raça & $-1,104$ & $0,047^{*}$ & 0,331 & 0,111 & 27,166 \\
Ocupação & 1,320 & 0,192 & 3,742 & 0,515 & 0,932 \\
Sobrepeso/Obesidade & $-1,101$ & $0,036^{*}$ & 0,333 & 0,119 & 10,561 \\
Etilismo & 0,998 & 0,150 & 2,714 & 0,698 & - \\
Constante & 0,655 & 0,213 & 1,925 & - & 1,069 \\
& & Campina Grande & & & - \\
\hline & & & & \\
Sedentarismo & $-0,971$ & $0,067^{*}$ & 0,379 & 0,134 & - \\
Constante & 0,182 & 0,670 & 1,200 & - & \\
\hline
\end{tabular}

Fonte: Base de dados da pesquisa realizada em 2011, Paes $(2008 ; 2009)$

$* \mathrm{p}<0,05$

OR: Odds Ratio

IC: Intervalo de Confiança

\section{DISCUSSÃO}

O perfil apresentado pelos homens do estudo favorece ao não controle da pressão, o que requer dos profissionais de saúde uma maior intensidade de ações focadas nos fatores de risco modificáveis. Na atualidade é comum encontrar homens com grande risco cardiovascular uma vez que apresentam um perfil de fumantes, etilistas, com altas taxas de colesterol ${ }^{11}$.

Estudos revelam a necessidade do estabelecimento de programas para rastrear precocemente a hipertensão, de terapias eficazes para a redução dos níveis pressóricos tanto sistólicos, como diastólicos ${ }^{12-13}$.Todavia estudos apontam que a utilização dos serviços prestados não altera significativamente os níveis pressóricos ${ }^{15}$. O controle pressórico deve passar pela adesão ao tratamento e acompanhamento pelo Serviço de Saúde (SS), porém estudo revela que os indivíduos com PANC apresentaram percentuais mais satisfatórios para adesão/ vínculo ${ }^{7}$. Essa situação pode ser explicada pela falta de adesão por parte dos hipertensos aos esquemas terapêuticos e à orientação inadequada fornecida pelos profissionais de saúde ${ }^{16-17}$. Autores destacam que pacientes com HAS detêm um bom conhecimento sobre a doença $(70,0 \%)$ e seu tratamento $(62,1 \%)$, porém um baixo controle da hipertensão $(29,6 \%)^{14}$.

A pressão arterial dos homens estudados não estava controlada, corroborando com outros estudos ${ }^{15}$. Para se conseguir o controle da PA o acompanhamento é uma condição que deve ser adotada pelos profissionais que compõem as equipes de saúde, seja ele médico ou enfermeiro, todavia subtende-se pela presença majoritária de PANC que as ações e tratamentos ofertados aos homens hipertensos não estão sendo efetivos, e uma implicação 
dessa situação é o aumento no número de internações por HAS e suas complicações ${ }^{18-19}$. Autores destacam que o controle da HAS pode ser mais efetivo a partir da conscientização dos pacientes e de uma maior adequação do tratamento ${ }^{14}$.

Entende-se que, alguns homens, mesmo querendo comparecer aos SS, estão impedidos de tal feito, por motivos como acessibilidade geográfica, física ou pelo fator tempo. O Ministério da Saúde ${ }^{20}$ lista como causa para a baixa procura aos serviços a incompatibilidade de horários entre as unidades e suas atividades laborais. A implantação de horário noturno é estratégia operacional para sanar esse problema $^{21}$. Porém, só isso não basta, pois é necessário que haja uma boa relação entre usuário e SS. A forma como as pessoas interagem com o sistema de atenção as conduz a decidirem sobre o quanto, como e o que utilizar deste sistema ${ }^{22}$.

Quando o vínculo não é acentuado, o estímulo familiar leva o homem a buscar o SS. A maioria dos sujeitos da pesquisa convive com alguém e este alguém assume o papel de cuidador. A presença permanente de uma companheira é um grande aliado no dia-a-dia, além de servir como suporte emocional ${ }^{23}$.

Outro fator relevante para o controle da PA é o nível de escolaridade. Nos dois municípios o alto nível de escolaridade foi mínimo, o que leva a dificuldade na interpretação e assimilação das informações. Estudos destacam que o maior número de analfabetos brasileiros tem 65 anos ou mais e possuem baixa renda e que a prevalência de HA é aumentada em indivíduos com menos de 4 (quatro) anos de escolaridade, geralmente decorrente da falta de conhecimento dos fatores de risco e do déficit em atividades preventivas ${ }^{16-24}$. Além disso, indivíduos com baixa escolaridade, etilistas e fumantes apresentam uma maior ingestão diária de $\mathrm{sal}^{25}$.

Nos dois municípios se destacou como mais prevalente o grupo não branco, reforçando os achados de outros estudos ${ }^{24-26}$, em que a hipertensão foi duas vezes mais em indivíduos pretos/pardos que na branca, situação respaldada na genética, pela afirmativa que estas pessoas apresentam elevados níveis de vasopressina plasmática, um maior declínio da resposta renal.

Destaca-se no estudo que menos da metade dos hipertensos estão inseridos no mercado de trabalho, por ocupação formal ou não, mantendo-os mais afastados dos SS e de práticas preventivas. Autores ${ }^{17-27}$ destacam como razões para os homens não irem aos serviços de saúde a falta de tempo, impossibilidade de deixar as atividades, ou medo da relevância do problema de saúde afetar o posto de trabalho. A renda contabilizada nos dois municípios foi > dois salários mínimos, destinada tanto para o sustento da família, como para dar suporte ao tratamento. Os serviços públicos de saúde assumem grande importância no tratamento dos hipertensos, já que o baixo poder aquisitivo não lhes dá acesso a planos particulares e limita a capacidade de manter sua saúde com a aquisição de alimentação adequada, acesso ao lazer, a prática de atividade física e o acesso ao $\mathrm{SS}^{18-28}$.

O grupo etário predominante do estudo foi o dos idosos (>60 anos) reforçando outros estudos. O aparecimento da HAS nesse grupo decorre da redução da complacência dos vasos, do enrijecimento das artérias e da diminuição de sua elasticidade, que associado a outros aspectos fisiopatológicos comprometem a função circulatória ${ }^{7-16}$. Na velhice, confrontados com a própria vulnerabilidade, os homens buscam mais os SS e apresentam quadros irreversíveis, resultante da falta de ações preventivas ou de tratamento precoce ${ }^{20}$.

A HAS mantém relação direta com fatores de risco modificáveis como tabagismo, etilismo, sedentarismo e sobrepeso/obesidade. O homem, enquanto ser que se acha invulnerável, os utiliza até como reforço da sua masculinidade. Os homens hipertensos atendidos na atenção primária em JP e CG na sua maioria não eram etilistas, tabagistas ou sedentários, todavia em CG a maioria era sedentária.

Os que se declararam não tabagistas e não etilistas, em grande proporção, já foram um dia, por um período superior a 15 anos e início em idade precoce (<15anos) inferior a 18 anos, época que não se tem maturidade e se sofre a influência de determinantes como moda, propagandas, amigos, familiares, entre outros.

O consumo de cigarro provoca efeitos impactantes na saúde (atinge todos os órgãos 
e altera suas funções) e na esperança de vida, por isso quando mais cedo se abandona essa prática mais cedo acabam seus efeitos deletéricos ${ }^{29}$. Dados do Instituto Nacional do Câncer ${ }^{30}$ apontam que o tabagismo é a segunda causa de morte no mundo, e que decorrente das ações de combate ao tabagismo o Brasil, de 1989 (35\%) para o ano 2008 (17,2\%), apresentou uma queda de $49,1 \%$ de fumantes na população de 15 anos.

No tocante ao uso do álcool, tanto JP como CG apresentaram baixos índices, apesar dele ser utilizado como promotor de relações sociais, presente em cerimônias, datas comemorativas e encontros. Autores ${ }^{28}$ evidenciaram que o álcool tem associação com a HA com um Odds Ratio (OR) de 2,94. Seu uso por períodos prolongados eleva a PA e a mortalidade por DCV, e a redução do seu consumo diminui, em média, os níveis da PAS em 3,3 mmHg e da PAD em 2,0 $\mathrm{mmHg}^{31}$.

O sedentarismo e sobrepeso nos homens do estudo podem justificar a presença da PANC nos dois municípios. Em JP essas variáveis foram menos incidentes, provavelmente pela existência de praias, atrativo para atividades físicas como caminhar, pedalar, nadar, dentre outras. Em CG, com valores maiores, pode-se justificar pelo clima (chuvas frequentes e frio), que afasta os hipertensos das atividades físicas ao ar livre. Convém ressaltar que a mortalidade e o risco de Doenças Cardiovasculares (DCV) é duas vezes maior em sedentários e que o exercício aeróbico é capaz de reduzir os níveis sistólicos em $7 \mathrm{mmHg}$ e os diastólicos em $5 \mathrm{mmHg}{ }^{29}$.

\section{CONCLUSÃO}

As variáveis raça e sobrepeso/obesidade mantiveram relação estatisticamente significativa com o controle da pressão. Com isso pode-se inferir que homens brancos, não sedentários ou que não se encontravam com sobrepeso/obesidade tem mais chance de controlar os níveis pressóricos quando comparados aos homens em situação contrária.

As principais limitações do estudo incluem ausência de análise bioquímica sanguínea,
Os fatores de risco modificáveis não são combatidos isoladamente e se conjuntamente. As VI Diretrizes Brasileiras de Hipertensão ${ }^{15}$ recomendam a adoção de medidas para manter o peso adequado, reduzir o consumo de sal, controlar o consumo de gorduras, incluir nas refeições alimentos ricos em potássio, praticar regularmente exercícios aeróbicos, moderar a ingestão de álcool, abandonar o tabagismo e controlar o estresse emocional. A partir dessa premissa se fortalece a necessidade do estabelecimento de programas de promoção da saúde e do fortalecimento do sistema de atenção primária à saúde ${ }^{32}$.

As variáveis definidas pelo modelo de regressão logística $(M R L)$ que tiveram relação significativa com o controle da PA foram a raça e o sobrepeso, no município de JP. O OR revela que indivíduos da raça branca e não obesos têm três vezes mais chance de controlar a pressão do que os indivíduos não brancos e os obesos. No modelo do município de CG apenas o sedentarismo, apesar da associação da mesma com o controle da PA ter sido menos significativo. Este achado encontra respaldo em outro trabalho ${ }^{29}$ onde seus autores afirmam que a mortalidade e o risco de DCV são duas vezes maiores nas pessoas sedentárias, e a prática de exercícios físicos em hipertensos e em préhipertensos é capaz de reduzir os valores das PAS e PAD.

O Intervalo de Confiança do OR a 95\% $(0,134$ - 1,069) revela que os indivíduos analisados apresentam chances iguais para o controle ou descontrole da pressão.

coleta de hormônios envolvidos com o desenvolvimento da HAS e histórico paternal de DCV. Assim, os dados apresentados em nosso estudo indicam dificuldade do controle da pressão, isso solicita dos profissionais de saúde intensificação de ações focadas nos fatores de risco estudados.

A divulgação desses resultados contribui para maior direcionamento dos fatores de risco na prevenção e tratamento da HAS. 


\section{REFERÊNCIAS}

1. Ferreira SRG, Moura EC, Malta DC, Sarno F. Frequência de hipertensão arterial e fatores associados: BRASIL, 2006. Rev Saúde Pública. 2009; 43(Supl 2), 98-106.

2. Yang G et al. Rapid health transition in China, 1990-2010: findings from the Global Burden of Disease Study 2010. The lancet. 2013 Jun 8; 381(9882):1987-2015.

3. Hiramoto JS, Katz R, Weisman S, Conte M. Gender-specific risk factors for peripheral artery disease in a voluntary screening population. J Am Heart Assoc. 2014 Mar; 13;3(2):e 000651.

4. Sociedade Brasileira de Cardiologia (SBC), Sociedade Brasileira de Hipertensão (SBH), Sociedade Brasileira de Nefrologia (SBN). VI Diretrizes Brasileiras de Hipertensão. Arq Bras Cardiol 2010; 95(1 supl.1): 1-51.

5. Brasil. Ministério da Saúde. Secretaria de Atenção à Saúde. Departamento de Atenção a Saúde. Hipertensão arterial sistêmica para o Sistema Único de Saúde. Ministério da Saúde, 2006.

6. Shapiro, D. J., Hersh, A. L., Cabana, M. D., Sutherland, S. M., \& Patel, A. I.Hypertension screening during ambulatory pediatric visits in the United States, 2000-2009. Pediatrics.2012; 130(4), 604-610.

7. Silva CS. Análise da dimensão adesão/vínculo dos hipertensos com níveis pressóricos não controlados nas Unidades de Saúde da Família do município de João Pessoa. [dissertação]. João Pessoa (PB):Universidade Federal da Paraíba; 2011.

8. Paes, NA. Avaliação da Efetividade no controle da hipertensão arterial sistêmica e associação com fatores de risco comparando a atenção do Programa de Saúde da Família e de Unidades Básicas de Saúde de municípios do Nordeste do Brasil. Edital: MCT/CNPq/ MS - SCTIE - DECIT/MS No. 37/2008 - Processo No. 576671/2008-8, 2008.

9. Sociedade Brasileira de Cardiologia, \& Sociedade Brasileira de Nefrologia. V Diretrizes brasileiras de hipertensão arterial. Rev. bras. Hipertens. 2006; 13(4), 260-312.

10. Brasil. Conselho Nacional de Saúde. Comissão Nacional de Ética em Pesquisa - CONEP. Portaria no 466/12 de Dezembro de 2012. Trata das diretrizes e normas regulamentadoras de pesquisas envolvendo seres humanos. Brasília; 2012.

11. Zatu MC, Van Rooyen JM, Loots DT, Greeff M, Schutte AE.A comparison of the cardiometabolic profile of black South Africans with suspected non-alcoholic fatty liver disease (NAFLD) and excessive alcohol use. Alcohol. 2015; 49(2), 165-172.

12. Bagyura Z et al. Cardiovascular screening programme in the Central Hungarian region. The Budakalász Study. Orvosi hetilap. 2014; 155(34), 1344-1352.

13. Rapsomaniki E et al. Blood pressure and incidence of twelve cardiovascular diseases: lifetime risks, healthy life-years lost, and agespecific associations in 1.25 million people. The Lancet. 2014 May 31;383(9932):1899-911.

14. Jiang B, Hongmei LIU, Xiaojuan RU, Zhang H, Shengping WU, Wenzhi WANG. Hypertension detection, management, control and associated factors among residents accessing community health services in Beijing. Scientific reports. 2014; 4.

15. Andrade FA. Comparativo dos níveis pressóricos sistêmicos e associação dos fatores de risco entre hipertensos segundo os critérios de acompanhamento em Unidades de Saúde da Família do município de João Pessoa-Pb. [dissertação]. João Pessoa (PB): Universidade Federal da Paraíba; 2011.

16. Sajuliani AF. Pré-hipertensão e risco cardiovascular global. Rev Bras Hipertens vol. 2009; 16(2):103-107.

17. Dias EM, Pereira RMN, Cavalcante HS, Ramalheiro NS, Silva NF, Carvalho Junior MR. Perfil epidemiológico dos pacientes com hipertensão arterial sistêmica cadastrados na Casa Saúde da Família Águas Lindas II, Belém, PA. Revista de Medicina. 2009 Jul.-dez; $88(3 / 4): 191-8$

18. Monteiro PC, Santos FS, Fornazari PA, Cesarino CB. Características biossociais, hábitos de vida e controle da pressão arterial dos pacientes em um programa de hipertensão. Arq Ciênc Saúde. 2005;12(2), 73-79.

19. Sousa LB, Sousa RKT, Scochi MJ. Hipertensão arterial e saúde da família: atenção aos portadores em município de pequeno porte na região sul do Brasil. Arq Bras Cardiol. 2006; 87(1)

20. Ministério da Saúde (BR). Secretaria de Atenção à Saúde. Departamento de Ações Programáticas Estratégicas. Política Nacional de Atenção Integral à saúde do Homem: princípios e diretrizes. Brasilia: Ministério da Saúde; 2008.

21. Santos FA. Dinâmica da acessibilidade masculina ao Programa de Saúde da Família. [dissertação] 126 f. Jequié (BA): Universidade Estadual do Sudoeste da Bahia; 2010.

22. Starfield B. Atenção primária: equilíbrio entre necessidades de saúde, serviços e tecnologia. Brasília: UNESCO, Ministério da Saúde; 2002.

23. Gravina CF, Grespan SM, Borges JL. Tratamento não-medicamentoso da hipertensão no idoso. Rev bras hipertens. 2007;14(1), 33-6.

24. Costa JSDD, Barcellos FC, Sclowitz ML, Sclowitz IKT, Castanheira M, Olinto MTA et al. Prevalência de hipertensão arterial em adultos e fatores associados: um estudo de base populacional urbana em Pelotas, Rio Grande do Sul, Brasil. Arquivos Brasileiros de Cardiologia. 2007; 88 (1):59-65.

25. Hong LJ. et al. Dietary sodium intake and its impact factors in adults of Shandong province. Biomedical and Environmental Sciences. $2014 ; 27(7), 564-566$.

26. Magnabosco P. Qualidade de vida relacionada à saúde do indivíduo com hipertensão arterial integrante de um grupo de convivência. [dissertação]. Ribeirão Preto (SP):Escola de Enfermagem de Ribeirão Preto da USP; 2007.

27. Schraiber L B, Gomes R, Couto MT. Homens e Saúde na Pauta da Saúde Coletiva. Revista Ciências \& Saúde. 2005; 10(1): 7-17.

28. Magalhães MEC, Brandão AA, Pozzan R, Campana EMG, Fonseca FL, Pizzi OL, Brandão AP. Prevenção da hipertensão arterial: para quem e quando começar. Rev Bras Hipertens. 2010; 17(2), 93-97.

29. Nunes E. Consumo de tabaco. Efeitos na saúde. Rev Port Clin Geral. 2006; 22(2).

30. Ministério da Saúde (BR). Instituto Nacional do Câncer - INCA. Observatório da política Nacional de controle do Tabaco. 2011. 31. Martins MSAS, Ferreira MG, Guimarães LV, Viana LAC. Hipertensão Arterial e Estilo de Vida em Sinop, Município da Amazônia Legal. Arq Bras Cardiol. 2010; 94(5), 639-44

32. Acharyya T, Kaur P, Murhekar MV. Prevalence of behavioral risk factors, overweight and hypertension in the urban slums of North 24 Parganas District, West Bengal, India, 2010.Indian journal of public health. 2014; 58(3), 195.

Recebido em 25 de novembro de 2015. Aprovado em 16 de maio de 2016. 